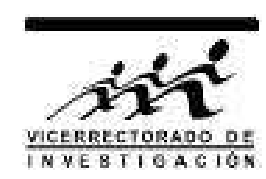

\title{
Esparcimiento de ondas electromagnéticas por microesferas dieléctricas
}

\author{
W. M. Solano-Reynoso*1 y J. Rojas Acuña ${ }^{2}$ \\ ${ }^{1}$ Laboratorio de Teledetección y Energías Renovables, Universidad Nacional de San Cristóbal de Huamanga, \\ Ayacucho, Perú \\ ${ }^{2}$ Facultad de Ciencias Físicas, Universidad Nacional Mayor de San Marcos, Lima, Perú
}

Recibido 25 noviembre 2016 - Aceptado 15 diciembre 2016

\begin{abstract}
La interacción del campo electromagnético con una esfera dieléctrica homogénea e isotrópica no magnetizada es analizada bajo la solución de Mie de las ecuaciones de Maxwell, considerando la absorción y el scattering para una longitud de onda variable de las ondas electromagnéticas respecto a las dimensiones de las microesferas.

Se muestran los resultados analíticos de los factores de extinción para diferentes índices de refracción que corresponden a diferentes materiales encontrados en la literatura y la determinación del espesor óptico de las partículas esféricas.
\end{abstract}

Palabras claves: Scattering, teoría de Mie, espesor óptico.

\section{Scattering of electromagnetic waves from dielectric microspheres}

The interaction of the electromagnetic field with a homogeneous and non-magnetized isotropic dielectric sphere is described under the Mie solution of Maxwell equations, considering the absorption and scattering for a variable wavelength of electromagnetic waves with respect to the microsphere sizes.

Results of the extinction factors for different refractive indices of different materials found in the literature and the determination of the optical thickness for spherical particles are shown.

Keywords: Scattering, Mie theory, optical thickness.

La descripción de la interacción de una onda electromagnética plana y polarizada con una esfera dieléctrica es un problema que se ha enfrentado desde el siglo XIX en relación al fenómeno de la difracción y el scattering. Si bien existen textos básicos sobre la interacción del campo electromagnético con partículas, genéricamente denominado scattering electromagnético [12], hay publicaciones más especializadas orientadas al scattering de la luz con partículas esféricas como la teoría de Mie y otras donde las partículas no son esféricas pero tienen soluciones analíticas, como el de van de Hulst [3], que es un texto completo, el de Kerker [4] que es de referencia primaria, y el texto completo con bastante información de Bohren y Huffman [5].

Lorenz publicó en danés en 1890, la solución del scattering por una esfera [6] sin tomar en cuenta las ecuaciones de Maxwell. Gustav Mie resuelve en 1908 [6] el scattering por una esfera utilizando las ecuaciones de Maxwell, en tanto que Debye en 1909 trata el problema relacionado a la presión de radiación sobre una partícula esférica utilizando funciones potenciales similares a la de Mie [6]. Si bien en la literatura se encuentran publicaciones con las denominaciones de teoría de Lorenz-Mie, teoría de Mie-Debye o teoría de Lorenz-Mie-Debye, porque el punto de partida de Mie fueron las soluciones a las ecuaciones de Maxwell, por ello gran parte de los autores en la literatura denominan simplemente solución de Mie o teoría de Mie, éste último término utilizaremos en el presente artículo.

Desde la segunda mitad del siglo $X X$ las soluciones basadas en la teoría de Mie comenzaron a utilizarse en la interacción de la radiación electromagnética con la atmósfera, existiendo ahora publicaciones al respecto [7.-9], especialmente referidas a los aerosoles atmosféricos [10,11] y atmósferas planetarias en astrofísica. Si bien nuestro interés primario son los aerosoles atmosféricos y su influencia en el forzamiento radiativo terrestre, es importante mencionar que la misma teoría tiene otras aplicaciones como en la microfísica de nubes. En las últimas décadas se ha incrementado el uso de la teoría de Mie en el estudio de materiales nanoestructurados hechos de nanopartículas sólidas aglomeradas [12]13].

*walmasolrey@gmail.com 
No obstante, hay que tener en cuenta que las partículas microscópicas donde se aplica la teoría de Mie no son totalmente esféricas, salvo en gotas de agua en nubes y otras situaciones muy especiales. Por ejemplo, en los aerosoles atmosféricos las partículas sólidas son amorfas en su geometría y no es posible encontrar soluciones analíticas, de modo que se utilizan métodos numéricos para resolver el problema que, en general, son computacionalmente pesados. En los últimos años se ha avanzado bastante en esta dirección habiéndose publicado textos al respecto como el de Mishchenko et. al. [14, 15, Doicu et. al. [16] y Rother y Kahnert [17], donde los cálculos numéricos están basados en las soluciones mediante las funciones de Green.

En este trabajo, presentamos la solución de Mie con bastante detalle para determinar analíticamente la absorción y el scattering producido por las micro esferas dieléctricas cuyo tamaño es del orden y menores que la longitud de onda de las ondas electromagnéticas incidentes.

\section{Teoría}

Se estudia un sistema conformado por una esfera dieléctrica de permitividad $\varepsilon$ sobre el cual incide un haz de radiación electromagnética con los campos mutuamente perpendiculares $\boldsymbol{E}_{\text {inc }}$ y $\boldsymbol{H}_{\text {inc }}$, de modo que se cumple la relación $\boldsymbol{B}=\mu \boldsymbol{H}$. Asimismo los campos inducidos dentro de la esfera son $\boldsymbol{E}_{\text {int }}$ y $\boldsymbol{H}_{\text {int }}$ y los haces esparcidos por la esfera muestran los campos $\boldsymbol{E}_{\text {sca }}$ y $\boldsymbol{H}_{\text {sca }}$ que juntamente con el haz incidente se progagan en un medio con permiti$\operatorname{vidad} \varepsilon_{1}$. En la Fig. 1 se muestra el esquema de los campos incidente y de scattering inelástico que produce absorción en la esfera.

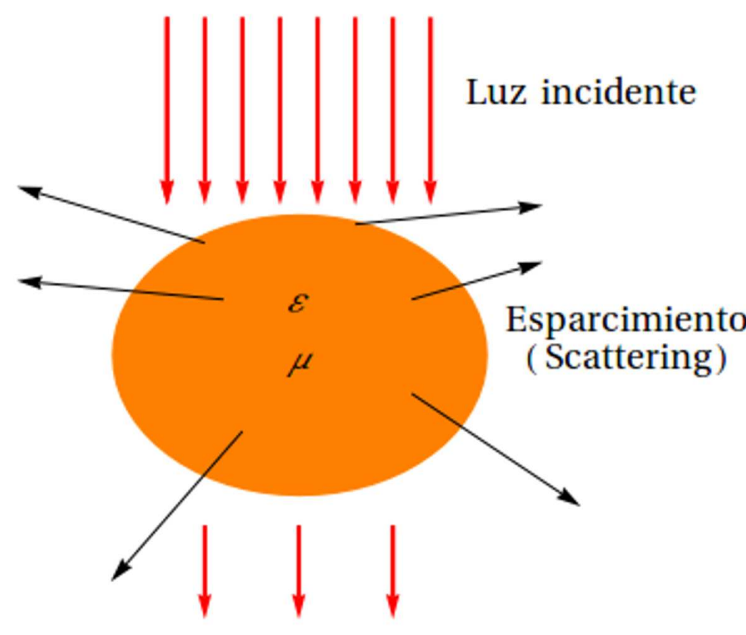

Luz directa atenuada

Figura 1: Esquema del scattering del campo electromagnético y un elipsoide.

La cuantificación de la energía transportada por la onda electromagnética por unidad de tiempo y unidad de superficie está dada por la media temporal del vector de Poynting, $\boldsymbol{S}=\boldsymbol{E} \times \boldsymbol{H}=\boldsymbol{E} \times \boldsymbol{B} / \mu$, para un período de la onda denotada como $\langle S\rangle$, de modo que la potencia viene a ser

$$
W=\int_{\Sigma}\langle\boldsymbol{S}\rangle \cdot \hat{\boldsymbol{n}} d \Sigma
$$

donde $\Sigma$ denota la superficie atravesada por el campo electromagnético y $\hat{\boldsymbol{n}}$ es el vector unitario perpendicular a la superficie. En el proceso de interacción del campo con la esfera se produce la extinción del campo electromagnético a través del scattering y la absorción por la partícula [5], que nos permite escribir la potencia extinguida como

$$
W_{\mathrm{ext}}=W_{\mathrm{sca}}+W_{\mathrm{abs}},
$$

en la que la potencia por scattering $W_{\text {sca }}$ se determina mediante la integral (1) usando para el vector de Poynting los campos de scattering, mientras que la potencia absorbida $W_{\text {abs }}$ se determina con los campos en el interior de la esfera.

\section{Secciones eficaces de scattering y factores de ex- tinción}

Se considera que la energía de la radiación que incide perpendicularmente a un área $\delta S^{\prime}$ está dado por $\delta E$, la cual es extinguida por el scattering y la absorción en la cantidad $\delta_{\text {ext }}$, definiendo la sección eficaz de extinción [9] como

$$
C_{\mathrm{ext}}=\frac{\delta E_{\mathrm{ext}}}{\delta E} \delta S^{\prime}
$$

Por conservación de energía, la relación entre las secciones eficaces de extinción, scattering y absorción está dado por

$$
C_{\text {ext }}=C_{\text {sca }}+C_{\text {abs }} .
$$

En la práctica estas secciones se calculan dividiendo la potencia de extinción entre la potencia incidente por unidad de área, $I_{0}$, o sea,

$$
C_{\mathrm{ext}}=\frac{W_{\mathrm{ext}}}{I_{0}}
$$

con relaciones similares para las secciones eficaces de scattering y absorción. Los conceptos definidos hasta ahora no dependen de la forma de la esfera. Si $A$ es el área de la proyección de la esfera sobre el plano perpendicular a la dirección de propagación del haz incidente [10], se define el factor de eficiencia de extinción, scattering y absorción como

$$
Q_{\mathrm{ext}}=\frac{C_{\mathrm{ext}}}{A}, \quad Q_{\mathrm{sca}}=\frac{C_{\mathrm{sca}}}{A} \quad \text { y } \quad Q_{\mathrm{abs}}=\frac{C_{\mathrm{abs}}}{A},
$$

respectivamente. En el caso de las esferas, $A$ se toma como el área del círculo. 


\section{Función de fase de scattering}

Puesto que la radiación es desviada en todas direcciones, hay que conocer el scattering en alguna dirección especificada. Si $d E_{\text {dir }}(r)$ es la energía en la dirección $r$, su valor es proporcional al ángulo sólido $d \Omega$ en dicha dirección y se define [9] la sección transversal de scattering direccional como

$$
C_{\mathrm{dir}}=\frac{d E_{\mathrm{dir}}}{I_{0} d \Omega}
$$

Queda claro que la integral de $d E_{\mathrm{dir}}(r)$ sobre todo el ángulo sólido resulta la sección eficaz de scattering $C_{\text {sca }}$ Similar a la definición de los factores de eficiencia, conviene definir la cantidad adimensional denominada función de fase de scattering [9] dada como

$$
\Phi(r)=4 \pi \frac{C_{\mathrm{dir}}(r)}{C_{\mathrm{sca}}},
$$

donde el factor $4 \pi$ se coloca por razones de normalización tal que

$$
\frac{1}{4 \pi} \int_{4 \pi} \Phi(r) d \Omega=1
$$

La dirección de scattering está determinada por el ángulo de scattering $\theta$ y el acimut de scattering $\phi$, es decir, $\Phi=\Phi(\theta, \phi)$. No obstante, en muchas aplicaciones, especialmente en aerosoles atmosféricos, la función de fase de scattering no depende del acimut, de modo que escribimos

$$
\frac{1}{2} \int_{0}^{\pi} \Phi(\theta) \operatorname{sen} \theta d \theta=1,
$$

La función de fase para esferas se puede conocer explícitamente.

\section{Distribución de tamaño de las esferas}

La extinción producida por una esfera se puede generalizar a un conjunto de esferas. Si hay $N$ esferas idénticas, la extinción producida será la suma, es decir, $C=N C_{\text {ext }}$. En caso de haber $N_{i}$ esferas idénticas por cada especie o tipo, entonces, el coeficiente de extinción es $C=\sum_{i} N_{i} C_{\text {ext }}$. Sin embargo, la descripción que mejor se aproxima a la situación real nos lleva a introducir el concepto de distribución de tamaño de las esferas con radios entre $r$ y $r+d r$, dada como

$$
n(r)=\frac{d N(r)}{d r}
$$

donde $N(r)$ es el número de esferas por unidad de volumen y $n(r)$ es la número de esferas por unidad de volumen con radios entre $r$ y $r+d r$. De entre las diversas formas de distribuciones de tamaño de las esferas [18,-20, una de las más utilizadas en el estudio de aerosoles atmosféricos es la distribución logarítmica normal denominada más comunmente como lognormal, dada como

$$
n(r)=\frac{N_{0}}{\sqrt{2 \pi}} \frac{1}{r \sigma} \exp \left[-\frac{(\ln r-\mu)^{2}}{2 \sigma^{2}}\right] .
$$

También se ha encontrado que muchas distribuciones son explicadas como distribuciones bimodales o multimodales, tal como a continuación escribimos para el caso de una distribución bimodal lognormal,

$$
\begin{array}{r}
n(r)=\frac{N_{1}}{\sqrt{2}} \frac{1}{r \sigma_{1}} \exp \left[-\frac{\left(\ln r-\mu_{1}\right)^{2}}{2 \sigma_{1}^{2}}\right]+ \\
\frac{N_{2}}{\sqrt{2}} \frac{1}{r \sigma_{2}} \exp \left[-\frac{\left(\ln r-\mu_{2}\right)^{2}}{2 \sigma_{2}^{2}}\right] .
\end{array}
$$

\section{Espesor óptico}

Cuando la radiación electromagnética atraviesa un sistema de muchas partículas microesféricas, ella es atenuada y su cuantificación se hace en términos de cantidades radiativas. Se ha descrito la extinción de la radiación incidente sobre una esfera y puede generalizarse considerando un sistema de partículas microesféricas donde incide el campo electromagnético. La pérdida de la energía incidente genéricamente se denomina atenuación y su cuantificación se describe mediante el concepto de espesor óptico. En la Fig. 2(a) se tiene el esquema de un sistema de partículas microesféricas donde incide las ondas electromagnéticas la que es atenuada por scattering y absorción, produciendo lo que se denomina la extinción $\varkappa=\lambda \alpha / 4 \pi$.

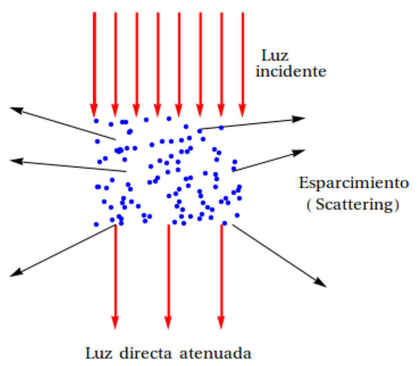

(a)

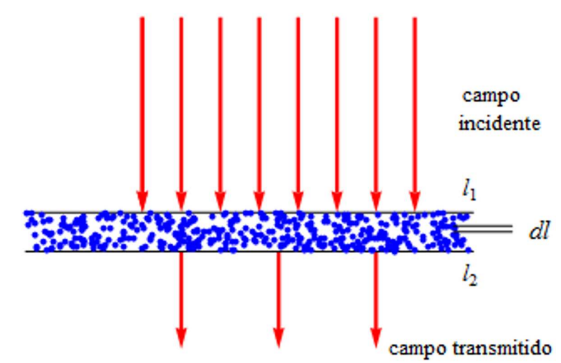

(b)

Figura 2: (a) Scattering por un sistema de partículas. (b) Esquema para la definición del espesor óptico. 
En la Fig. 2(b) se considera que la radiación incidente tiene una intensidad $I_{\lambda}$ que atraviesa perpendicularmente el espesor $d l$, entonces, la fracción $d I_{\lambda}$ de energía atenuada respecto a la incidente es proporcional al ancho $d l$ que atraviesa, escribiendo

$$
\frac{d I_{\lambda}}{I_{\lambda}}=-\alpha_{\lambda} d l
$$

donde $\alpha_{\lambda}$ se denomina coeficiente de volumen de extinción [7,9]. Para el caso que nos interesa, por conservación de energía se puede demostrar que

$$
\alpha_{\lambda \mathrm{ext}}=\alpha_{\lambda \mathrm{sca}}+\alpha_{\lambda \mathrm{abs}} .
$$

La integración de la ecuación (14) de un valor de referencia 0 a otro $l$, nos lleva a

$$
I_{\lambda}(l)=I_{\lambda}(0) \exp \left[-\tau_{\lambda \text { ext }}\right]
$$

donde

$$
\tau_{\lambda \text { ext }}=\int_{0}^{l} \alpha_{\lambda \text { ext }}\left(l^{\prime}\right) d l^{\prime} .
$$

La relación

$$
\frac{I_{\lambda}(l)}{I_{\lambda}(0)}=\exp \left[-\tau_{\lambda \text { ext }}\right]
$$

se denomina transmitancia, que es la fracción de energía incidente que pasa por el espesor $l$. Esta es una de las cantidades que pueden medirse experimentalmente. Es sencillo demostrar la relación entre el coeficiente de volumen de extinción y la sección eficaz de extinción [9], el cual es

$$
\alpha_{\lambda \text { ext }}=n C_{\text {ext }},
$$

donde $n$ es el número de microesferas por volumen. Teniendo en cuenta la distribución continua de tamaño de las microesferas y la relación entre la sección eficaz y el factor de eficiencia de extinción, escribimos para el espesor óptico

$$
\tau_{\lambda \mathrm{ext}}=\int_{0}^{\infty} A Q_{\mathrm{ext}}(r) n(r) d r .
$$

Si se trata de partículas esféricas se tiene $A=\pi r^{2}$.

\section{Los campos electromagnéticos en medios absor- bentes}

Como los campos eléctricos y magnéticos son armónicos en el tiempo, se usa la notación compleja para representarlos [5]. Así,

$$
\boldsymbol{E}(\boldsymbol{r}, t)=\boldsymbol{E}(\boldsymbol{r}) e^{-i \omega t}, \quad \boldsymbol{B}(\boldsymbol{r}, t)=\boldsymbol{B}(\boldsymbol{r}) e^{-i \omega t},
$$

de modo que las propiedades del medio donde se propaga la onda electromagnética satisfacen $\boldsymbol{D}=\varepsilon \boldsymbol{E}$ y $\boldsymbol{B}=\mu \boldsymbol{H}$, donde $\boldsymbol{E}$ representa el campo eléctrico, $\boldsymbol{D}$ es el desplazamiento eléctrico, $\boldsymbol{B}$ el campo de inducción magnética y $\boldsymbol{H}$ la intensidad magnética. Estos campos satisfacen las ecuaciones de Maxwell en medios materiales isotrópicos y homogéneos,

$$
\begin{aligned}
\nabla \cdot[\epsilon \boldsymbol{E}(\boldsymbol{r})] & =0, \\
\nabla \cdot[\mu \boldsymbol{H}(\boldsymbol{r})] & =0, \\
\nabla \times \boldsymbol{E}(\boldsymbol{r}) & =i \omega \mu \boldsymbol{H}(\boldsymbol{r}), \\
\nabla \times \boldsymbol{H}(\boldsymbol{r}) & =-i \omega \varepsilon \boldsymbol{E}(\boldsymbol{r}) .
\end{aligned}
$$

Se trata de resolver estas ecuaciones cuando sobre la esfera dieléctrica incide un haz de onda electromagnética que es esparcida por la esfera y a la vez absorbida. En este contexto, la permitividad dieléctrica es en general compleja [1.5] y, por lo tanto, se tendrá un índice de refracción complejo dado como

$$
\tilde{n}=n+i \varkappa,
$$

donde la parte real $n$ es el índice de refracción normal que usamos, mientras que la parte imaginaria $\varkappa$ es el coeficiente de absorción que tiene que ver con la absorción de la radiación [1,5], $\alpha=4 \pi \varkappa / \lambda$. Algunos autores [9, 22] prefieren usan la parte armónica en el tiempo como $e^{i \omega t}$, lo cual hace que el índice de refracción se escriba como $\tilde{n}=n-i \varkappa$.

Los campos $\boldsymbol{E}$ y $\boldsymbol{H}$ satisfacen las ecuaciones de Helmholtz vectoriales escritas como

$$
\boldsymbol{\nabla}^{2}+k^{2} \boldsymbol{E}=0, \quad \nabla^{2}+k^{2} \boldsymbol{H}=0, \quad k^{2}=\omega^{2} \varepsilon \mu .
$$

Es suficiente resolver una de estas ecuaciones para $\boldsymbol{E}(r)$ 。 $\boldsymbol{H}(r)$, ya que el otro campo se determina usando el rotacional de las ecuaciones (24) o (25). Como no existen cargas libres ni corrientes eléctricas, las condiciones de frontera que satisfacen los campos están dadas como

$$
\begin{aligned}
{\left[\boldsymbol{E}_{\mathrm{inc}}+\boldsymbol{E}_{\mathrm{sca}}-\boldsymbol{E}_{\mathrm{int}}\right] } & \times \hat{\boldsymbol{n}}=0, \\
{\left[\boldsymbol{H}_{\mathrm{inc}}+\boldsymbol{H}_{\mathrm{sca}}-\boldsymbol{H}_{\mathrm{int}}\right] } & \times \hat{\boldsymbol{n}}=0,
\end{aligned}
$$

donde $\hat{\boldsymbol{n}}$ es el vector unitario normal saliente a la superficie de separación entre la partícula y el medio que la rodea, los subíndices inc, sca y int denotan los campos incidente, scatterado e interior de la partícula, respectivamente. En principio las soluciones dependerán de la forma de la superficie frontera de la partícula.

\section{La solución de Mie.}

Para el caso de una partícula seguimos en gran parte el procedimiento dado por Bohren-Huffman [5], donde la idea es encontrar soluciones vectoriales de campos vectoriales $M$ y $N$ que satisfacen la ecuación vectorial de Helmholtz, para seguidamente expresar los campos eléctricos y magnéticos como combinaciones lineales de $\boldsymbol{M}$ y $\boldsymbol{N}$ y los coeficientes de la expansión se determinan usando las condiciones de frontera que deben satisfacer $\boldsymbol{E}$ y $\boldsymbol{H}$. Se define un campo vectorial $M$ como

$$
\boldsymbol{M}=\boldsymbol{\nabla} \times(\boldsymbol{r} \psi),
$$

que satisface la ecuación diferencial

$$
\nabla^{2} \boldsymbol{M}+k^{2} \boldsymbol{M}=\boldsymbol{\nabla} \times\left[\boldsymbol{r}\left(\nabla^{2} \psi+k^{2} \psi\right)\right],
$$


definiendo también otro vector $N$ como

$$
N=\frac{\nabla \times M}{k},
$$

que satisface la ecuación de Helmholtz

$$
\nabla^{2} \boldsymbol{N}+k^{2} \boldsymbol{N}=0 .
$$

Para que también $M$ se reduzca a la ecuación de Helmholtz, se observa en la ecuación (30) que debe satisfacerse

$$
\nabla^{2} \psi+k^{2} \psi=0
$$

con lo cual $\boldsymbol{M}$ y $\boldsymbol{N}$ satisfacen ecuaciones similares a la de los campos $\boldsymbol{E}$ y $\boldsymbol{B}$. Así, para determinar $\boldsymbol{M}$ y $\boldsymbol{N}$ es suficiente encontrar $\psi$ resolviendo la ecuación (33), que es una ecuación de Helmoholtz escalar, cuyas soluciones en coordenadas esféricas $(r, \theta, \phi)$ nos dan las funciones esféricas de Bessel $j_{n}$ y $y_{n}$ para la parte radial que denotamos como $z_{n}$, los polinomios asociados de Legendre $P_{n}^{m}(\cos \theta)$ para la parte angular $\theta$, y las funciones seno y coseno para la variable $\phi$, escribiendo las soluciones como

$$
\psi_{\text {emn }}(r, \theta, \phi)=\cos (m \phi) P_{n}^{m}(\cos \theta) z_{n}(k r)
$$

y

$$
\psi_{\text {omn }}(r, \theta, \phi)=\operatorname{sen}(m \phi) P_{n}^{m}(\cos \theta) z_{n}(k r)
$$

donde $\psi_{e m n}$ son las soluciones pares y $\psi_{\text {omn }}$ las soluciones impares. Con estas soluciones es fácil escribir las funciones vectoriales pares $\boldsymbol{M}_{e m n}(r, \theta, \phi), \boldsymbol{N}_{e m n}(r, \theta, \phi)$, e impares $\boldsymbol{M}_{\text {omn }}(r, \theta, \phi), \boldsymbol{N}_{\text {omn }}(r, \theta, \phi)$, los cuales son denominados armónicos esféricos vectoriales.

Las condiciones de frontera que satisfacen los campos eléctricos y magnéticos en coordenadas polares esféricas se dan como

$$
\begin{aligned}
& E_{\mathrm{inc} \theta}+E_{\mathrm{sca} \theta}=E_{\mathrm{int} \theta}, \\
& E_{\mathrm{inc} \phi}+E_{\mathrm{sca} \phi}=E_{\mathrm{int} \phi}, \quad r=a,
\end{aligned}
$$

y

$$
\begin{aligned}
H_{\mathrm{inc} \theta}+H_{\mathrm{sca} \theta} & =H_{\mathrm{int} \theta}, \\
H_{\mathrm{inc} \phi}+H_{\mathrm{sca} \phi} & =H_{\mathrm{int} \phi}, \quad r=a .
\end{aligned}
$$

Se toma el campo eléctrico incidente como un onda polarizada en la dirección $x$ dado como

$$
\boldsymbol{E}_{\text {inc }}=E_{0} e^{i k r \cos \theta} \hat{\boldsymbol{e}}_{x},
$$

que escrita en término de los armónicos esféricos vectoriales resulta

$$
\boldsymbol{E}_{\mathrm{inc}}=E_{0} \sum_{n=1}^{\infty} i^{n} \frac{2 n+1}{n(n+1)}\left(\boldsymbol{M}_{o 1 n}^{(1)}-i \boldsymbol{N}_{e 1 n}^{(1)}\right),
$$

donde el superíndice (1) denota que intervienen las funciones de Bessel $j_{n}$. Del mismo modo, se puede encontrar la expresión del campo scattered como

$$
\boldsymbol{E}_{\mathrm{sca}}=E_{0} \sum_{n=1}^{\infty} \frac{2 n+1}{n(n+1)}\left(i a_{n} \boldsymbol{N}_{e 1 n}^{(3)}-b_{n} \boldsymbol{M}_{o 1 n}^{(3)}\right)
$$

y el campo dentro de la esfera

$$
\boldsymbol{E}_{\mathrm{int}}=E_{0} \sum_{n=1}^{\infty} i^{n} \frac{2 n+1}{n(n+1)}\left(c_{n} \boldsymbol{M}_{\mathrm{o} 1 n}^{(1)}-i d_{n} \boldsymbol{N}_{\mathrm{e} 1 n}^{(1)}\right),
$$

donde el superíndice (3) denota las funciones esféricas de Hankel $h_{n}^{(1)}(r)$ [23]. Los coeficientes $a_{n}, b_{n}, c_{n}$ y $d_{n}$ se determinan usando las condiciones de frontera. Los dos primeros son de interés para nuestros cálculos, los cuales se escriben como

$$
\begin{aligned}
a_{n} & =\frac{\mu m^{2} j_{n}(m x)\left[x j_{n}(x)\right]^{\prime}-\mu_{1} j_{n}(x)\left[m x j_{n}(m x)\right]^{\prime}}{\mu m^{2} j_{n}(m x)\left[x h_{n}^{1}(x)\right]^{\prime}-\mu_{1} h_{n}^{(1)}(x)\left[m x j_{n}(m x)\right]^{\prime}} \\
b_{n} & =\frac{\mu_{1} m j_{n}(m x)\left[x j_{n}(x)\right]^{\prime}-\mu j_{n}(x)\left[m x j_{n}(m x)\right]^{\prime}}{\mu_{1} j_{n}(m x)\left[x h_{n}^{1}(x)\right]^{\prime}-\mu h_{n}^{(1)}(x)\left[m x j_{n}(m x)\right]^{\prime}}
\end{aligned}
$$

donde las primas denotan las primeras derivadas, $m$ denota el índice de refracción complejo de la esfera relativo al medio

$$
m=\frac{\tilde{n}}{\tilde{n}_{m}} \quad \text { y } \quad x=\frac{2 \pi a \tilde{n}_{m}}{\lambda}
$$

siendo $\tilde{n}$ el índice de refracción de la esfera, $\tilde{n}_{\mathrm{m}}$ del medio, $a$ el radio de la esfera y $\lambda$ la longitud de onda del campo incidente.

Las funciones $\psi(r)$ y $\xi(r)$ son las funciones de RiccatiBessel definidas como

$$
\psi(r)=r j_{n}(r), \quad \xi(r)=r h_{n}^{(1)}(r),
$$

donde las funciones de Hankel $h_{n}^{(1)}(r)$ facilitan la aproximación de la parte radial para grandes distancias. Así, el campo eléctrico de scattering a grandes distancias es aproximadamente transversal a la dirección de propagación, que se escribe como

$$
\begin{aligned}
\boldsymbol{E}_{\mathrm{sca}} \approx E_{0} \frac{e^{i k_{1} r}}{-i k_{1} r} & {\left[S_{2}(\cos \theta) \cos \phi \hat{\boldsymbol{e}}_{\theta}\right.} \\
- & \left.S_{1}(\cos \theta) \operatorname{sen} \phi \hat{\boldsymbol{e}}_{\phi}\right], \quad r \rightarrow \infty
\end{aligned}
$$

donde

$$
S_{1}(\cos \theta)=\sum_{n=1}^{\infty} \frac{2 n+1}{n(n+1)}\left[a_{n} \pi_{n} \cos \theta+b_{n} \tau_{n} \cos \theta\right]
$$

y

$$
S_{2}(\cos \theta)=\sum_{n=1}^{\infty} \frac{2 n+1}{n(n+1)}\left[a_{n} \tau_{n}(\cos \theta)+b_{n} \pi_{n}(\cos \theta)\right],
$$

siendo

$$
\pi_{n}(\cos \theta)=\frac{P_{n}^{1}(\cos \theta)}{\operatorname{sen} \theta}, \quad \tau_{n}(\cos \theta)=\frac{d P_{n}^{1}(\cos \theta)}{d \theta} .
$$


Con la ayuda de la Fig. 3 y la Ec. (46) se puede ver que el campo de scattering a grandes distancias está prácticamente polarizado en una dirección perpendicular al plano de scattering $\left(\hat{\boldsymbol{e}}_{\phi}\right)$ y otra contenida en el plano $\left(\hat{\boldsymbol{e}}_{\boldsymbol{\theta}}\right)$, de modo que $S_{1}$ está relacionado con la componente perpendicular y $S_{2}$ con la paralela al plano, que representan las funciones de fase de scattering del campo lejano de polarización perpendicular y paralela, respectivamente. Esto significa que el módulo al cuadrado de $\left|S_{1}\right|^{2}$ y $\left|S_{2}\right|^{2}$ son proporcionales a las respectivas intensidades de polarización, mientras que la suma representa la intensidad de la luz no polarizada. Es claro que el vector de onda de scattering, $\boldsymbol{k}_{s}$, se encuentra en el plano de scattering.

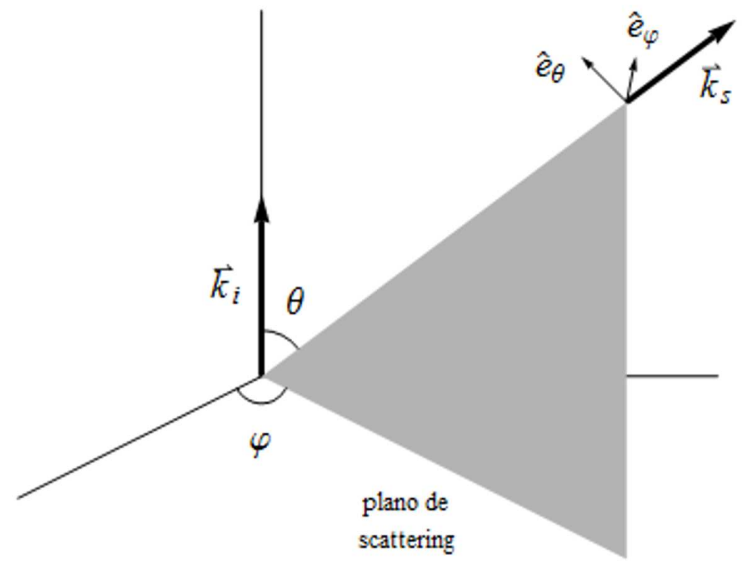

Figura 3: Campo eléctrico incidente y de scattering a grandes distancias, donde se muestra las dos direcciones de polarización, uno contenido en el plano de scattering y el otro perpendicular.

Así, la expresión de la intensidad de la función de fase no polarizada [24] es dada como

$$
\Phi(\cos \theta)=\frac{2}{x^{2} Q_{\text {sca }}}\left[\left|S_{1}(\cos \theta)\right|^{2}+\left|S_{2}(\cos \theta)\right|^{2}\right] .
$$

También tenemos los factores de eficiencia de scattering y extinción para partículas microesféricas,

$$
\begin{aligned}
Q_{\text {sca }} & =\frac{C_{\text {sca }}}{\pi r^{2}} \\
& =\frac{\lambda}{2 \pi N^{2}} \sum_{n=1}^{\infty}(2 n+1)\left(\left|a_{n}\right|^{2}+\left|b_{n}\right|^{2}\right) \\
& =\frac{2}{x^{2}} \sum_{n=1}^{\infty}(2 n+1)\left(\left|a_{n}\right|^{2}+\left|b_{n}\right|^{2}\right) .
\end{aligned}
$$

$$
\begin{aligned}
Q_{\text {ext }} & =\frac{C_{\text {ext }}}{\pi r^{2}} \\
& =\frac{\lambda}{2 \pi N^{2}} \sum_{n=1}^{\infty}(2 n+1) \operatorname{Re}\left\{a_{n}+b_{n}\right\} \\
& =\frac{2}{x^{2}} \sum_{n=1}^{\infty}(2 n+1) \operatorname{Re}\left\{a_{n}+b_{n}\right\} .
\end{aligned}
$$

Puesto que el albedo es la energía de scattering en una superficie, esta cantidad se puede cuantificar escribiendo el albedo como

$$
w=\frac{C_{\text {sca }}}{C_{\text {ext }}} .
$$

Una medida de la media por donde se da el scattering es el factor o parámetro de asimetría definido como

$$
\begin{aligned}
g=\langle g\rangle=\frac{4}{x^{2} Q_{\text {sca }}} \sum_{n=1}^{\infty}[ & \frac{n(n+2)}{n+1} \operatorname{Re}\left(a_{n} a_{n+1}^{*}+b_{n} b_{n+1}^{*}\right) \\
& \left.+\frac{2 n+1}{n(n+1)} \operatorname{Re}\left(a_{n} b_{n}^{*}\right)\right], \quad \text { (54) }
\end{aligned}
$$

donde $\theta$ es el ángulo de scattering respecto a la dirección de incidencia.

Muchos de los resultados mostrados no son sencillos de obtener, desde la aplicación de las condiciones de frontera existe muchos detalles que reúne decenas de páginas de arduo trabajo, que no se muestran detalladamente en las publicaciones por ello este proceso resulta ser un buen ejercicio para el lector interesado.

\section{Resultados y discusiones}

De la Ec. (52), los factores de eficiencia de extinción dependen del parámetro de tamaño $x$, Ec. (44), éste a su vez depende del radio de la esfera y la longitud de onda incidente. Generalmente se desea saber cómo es el comportamiento del factor de eficiencia cuando varía el radio y para ello nos valemos de las gráficas que nos darán información cualitativa, considerando que el medio es el vacío donde el índice de refracción es la unidad y la longitud de onda del campo incidente se mantiene constante, haciendo que en $x$ varíe sólo el radio de la microesfera. En la Fig. 4 se tiene $Q_{\text {ext }}(x)$ para gotas de agua con índice de refracción real 1.33 , que es una característica cualitativa similar para partículas con índices de refracción real. Se aprecia que hasta el primer máximo estamos en la región de radio de la partícula muy pequeño, es decir, el régimen aproximado de $x \ll 1$, donde el radio de la partícula es muy pequeña respecto a la longitud de onda y se demuestra que coincide con el scattering de Rayleigh [5] 25]. La región de las ondulaciones o máximos y mínimos corresponde a tamaños de la esfera del orden de la longitud de onda, donde ocurren los efectos de interferencia entre las ondas de scattering, cuyos detalles no son simples de explicar [25,26]. Las ondulaciones tienden a desaparecer y nos encontramos en la región 
de pequeñas longitudes de onda respecto al tamaño de la partícula, donde la óptica de rayos es suficiente para la descripción de la interacción [5 26], sin embargo, de acuerdo a la óptica de rayos la sección transversal de extinción debía ser del tamaño de la partícula y resulta el doble [27], algo que la óptica de rayos no puede explicar y en la literatura se conoce como la paradoja de extinción. En relación a esta paradoja, observar que la eficiencia de extinción oscila en torno del valor 2 a donde tiende justamente $Q_{\text {ext }}$.

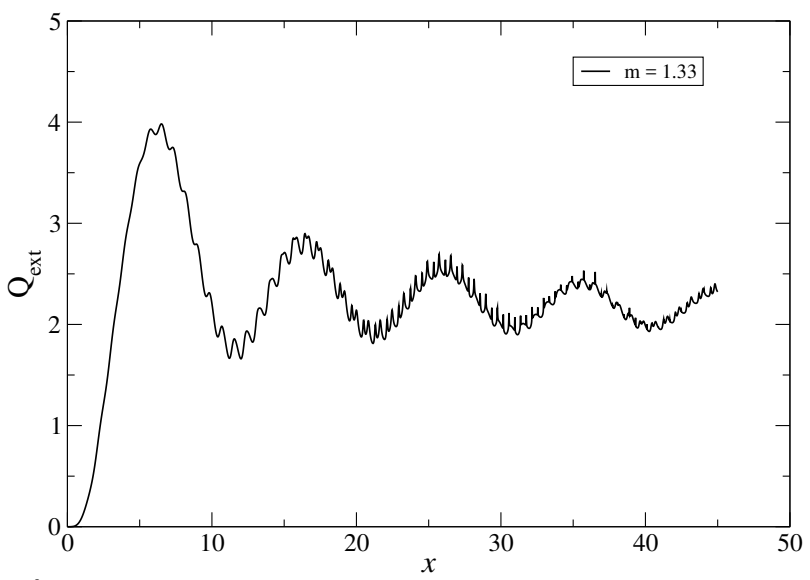

Figura 4: Comportamiento típico del factor de eficiencia de extinción $Q_{\text {ext }}$ en función del parámetro de tamaño $x$. En este caso no hay absorción de energía por la esfera.

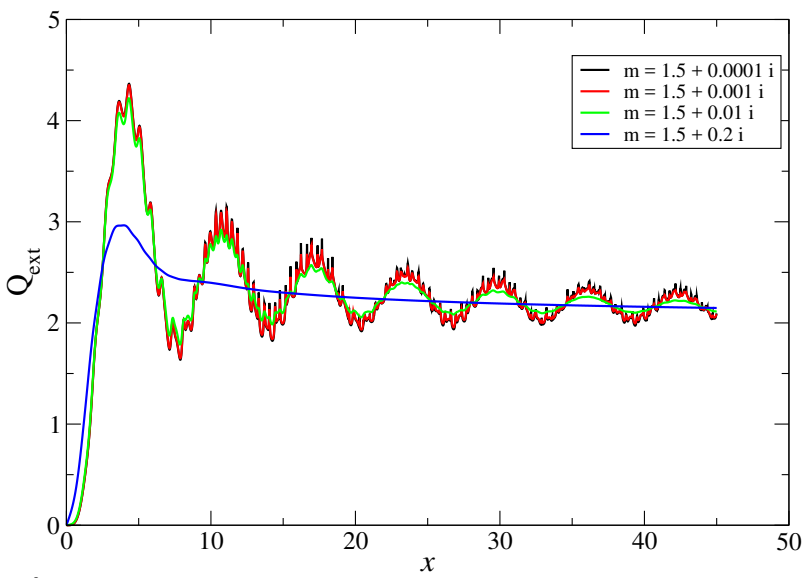

Figura 5: Eficiencia de extinción de una esfera absorbente para diferentes valores crecientes de la parte imaginaria del índice de refracción.

Ahora es interesante observar la eficiencia de extinción cuando los índices de refracción son complejos. En la Fig. 5 se grafica la eficiencia para una partícula cuya parte real del índice de refracción no cambia, pero sí la parte imaginaria. Las ondulaciones van disminuyendo o desapareciendo en la medida que se incrementa la parte imaginaria del índice de refracción. Este efecto se explica teniendo en cuenta que la parte imaginaria del índice de refracción está relacionada a la absorción de energía por parte del material, atenuando los efectos de scattering.

Observar que en el caso del índice de refracción $m=$ $1.5+0.2 i$ de la Fig. 5 (d), no se presentan las ondulaciones, esto significa que ocurre una fuerte absorción de energía por parte de esfera y un débil scattering de modo que se aprecia claramente que el factor de eficiencia de extinción tiende a 2.

En la Fig. 6 tenemos las eficiencias de extinción para tres valores del índice de refracción complejo, que reproduce el comportamiento de la atenuación de la onda cuando se incrementa la parte imaginaria del índice de refracción. En la Fig. 6(a) se ha tomado el índice de refracción real de 1.33 para la curva gris de mayor altura que presenta más ondulaciones, mientras que la curva punteada tiene la parte compleja de $0.05 \mathrm{i}$ y la curva continua sin ondulación tiene la parte compleja de $0.5 \mathrm{i}$. En la Fig. 6(b) se ha incrementado la parte real del índice de refracción a 3.0 cuyo efecto es incrementar la altura de las ondulaciones y la cantidad de picos, haciéndo más estrecha las ondulaciones. Esto corresponde a la hematita.

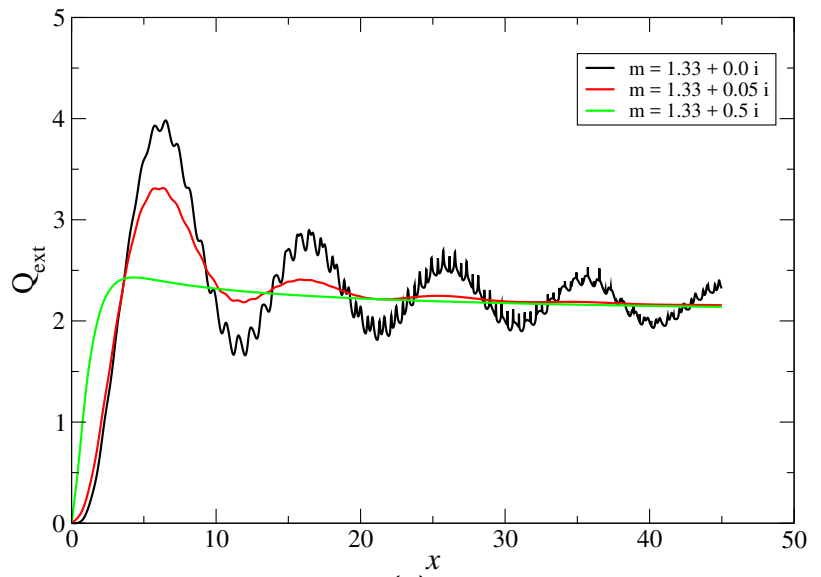

(a)

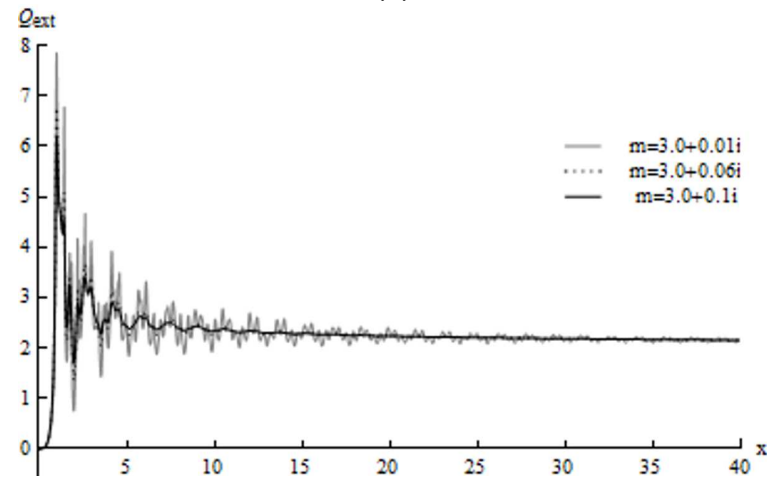

(b)

Figura 6: Se muestran las eficiencias de extinción para valores crecientes de la parte real e imagianria del índice de refracción. 
En la Fig. 7(a) tenemos el albedo de una partícula esférica dada por la Ec. (53), que se incrementa con el tamaño de la partícula, algo que intuitivamente debía ocurrir, si bien no de manera lineal. Observar que en la ordenada los valores del albedo parecieran ser constantes e iguales a la unidad. En realidad son muy próximos a la unidad y tenemos algunos valores para ilustrar la pequeña diferencia que hay entre ellos en función del parámetro de tamaño:

0.9999996946340548
0.9999998943389818
0.9999996946340548
0.9999999300695169
0.9999999401417841
0.9999999407829259
0.9999999341554375

con 16 dígitos de precisión con el que trabaja por defecto el programa Mathematica. El factor de asimetría dada por la Ec. (54) se muestra en la Fig. 7(b), donde el promedio del coseno del ángulo de scattering crece con el tamaño de la partícula esférica.

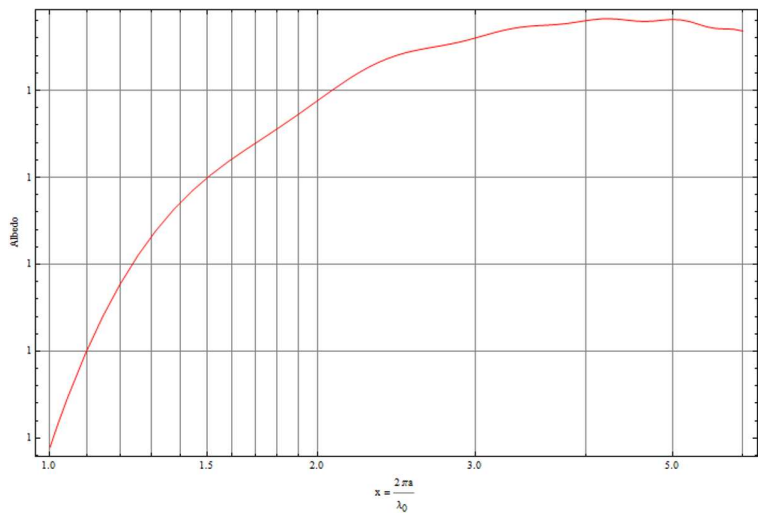

(a)

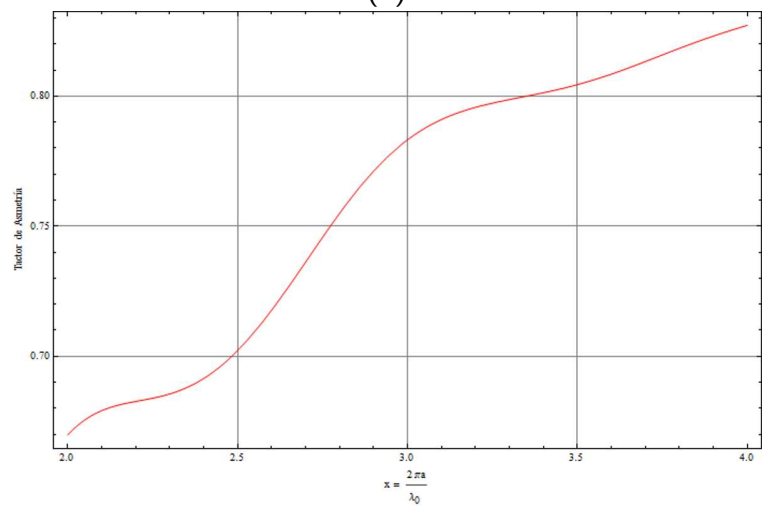

(b)

Figura 7: Se muestra (a) el albedo del scattering y (b) el factor de asimetría de una esfera.

Finalmente, en la Fig. 8 se muestra la intensidad de scattering con polarización perpendicular y paralela al plano de scattering, representadas por los módulos al cuadrado de las ecuaciones (47) (48) y (48)(49), respectivamente, mientras que la no polarizada es el promedio de las dos polarizaciones. Las tres últimas gráficas son adpataciones del código publicado por Lampado [28].

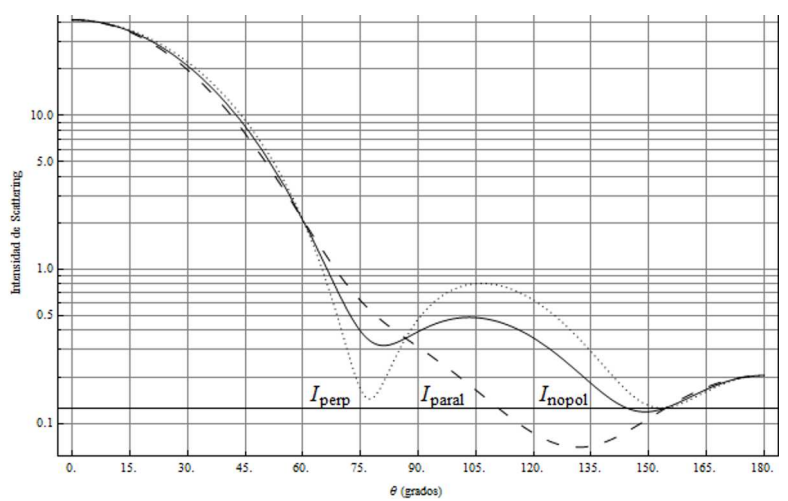

Figura 8: Se muestra las intensidades de scattering polarizadas y no polarizadas perpendicular y paralela al planos de scattering.

En los resultados mostrados se ha tomado el vacío como el medio donde está inmersa la esferaa, sin embargo, los resultados teóricos de la interacción de la radiación electromagnética con un partícula esférica son generales, en el sentido de que el medio puede ser de cualquier permitividad homogénea e isotrópica no absorbente. Una extensión para medios absorbentes se puede encontrar en las publicaciones de Fu y Sun [29,30].

\section{Conclusiones}

Se ha descrito la interacción de ondas electromagnéticas planas con una esfera de permitividad dieléctrica homogénea e isotrópica inmerso en un medio también homogéneo e isotrópico, que en nuestro caso tomamos el vacío. Históricamente, para el caso de una esférica se denomina teoría de Mie, donde en la descripción de la interacción se basa en la solución de las ecuaciones de Maxwell en medios materiales, siendo un tratamiento clásico y macroscópico. Los resultados encontrados explican físicamente la extinción de la energía de la onda electromagnética en su interacción con la esférica. Si bien en la naturaleza no todas las partículas son esféricas, la teoría de Mie es el punto de partida para entender cualitativamente la interacción del campo electromagnétco con una partícula, siendo una aplicación relevante para los estudios de los aerosoles atmosféricos y en las últimas décadas en la óptica de materiales nanoparticulados. También en los últimos veinte años se han tenido avances teóricos y experimentales en la óptica de la interacción con partícula no esféricas, cuyo punto de partida es necesariamente la teoría de Mie. Aún existiendo muchas publicaciones especializadas al respecto, esperamos que esta contribución sea de gran ayuda para quienes se inicien en los estudios de los aerosoles atmosféricos y en la óptica de materiales nanoparticulados. 


\section{Referencias}

[1] J. D. Jackson; Classical Electrodynamics, Third Edition, John Wiley \& Sons Inc., New York (1998).

[2] L. Tsang, J. A. Kong y K. H. Ding; Scattering of Electromagnetics Waves, Theories and Applications, John Wiley \& Son Inc., New York (2000).

[3] H. C. Van de Hulst; Light Scattering by Small Particles, John Wiley \& Sons Inc., Dover, New York (1981).

[4] Milton Kerker y Ernest M. Loebl; The Scattering of Light and Other Electromagnetic Radiation, Elsevier, Amsterdam (1969).

[5] C. F. Bohren y D. R. Huffman; Absorption and Scattering of Light by Small Particles, John Wiley \& Sons Inc., New York (1983).

[6] H. Wolfram y T. Wriedt; The Theory Mie, Basics and Applications, Springer, Berlin (2012).

[7] Jacqueline Lenoble; Atmospheric Radiative Transfer, A. Deepak Publishing (1993).

[8] K. N. Liou; An Introduction to Atmospheric Radiation, Second Ed., Academic Press, New York (2002).

[9] Y. M. Timofeyev y A.V. Vasielev; Theoretical Fundamentals of Atmospheric Optics, Cambridge International Science Publishing Ltd., Cambridge (2008).

[10] A. Kokhanovsky; Aerosol Optics, Light Absorption and Scattering by Particles in the Atmosphere, Springer \& Praxis, New York (2008).

[11] J. Lenoble, L. Remer y D. Tanré; Aerosol Remote Sensing, Springer, Praxis Publishing, New York (2013).

[12] S. Volz; Thermal Nanosystems and Nanomaterials, Springer, Berlin (2009).

[13] M. Quinten; Optical Properties of Nanoparticle Systems, Wiley-VCH, Berlin (2011).

[14] M. J. Mishchenko, J.W. Hovenier y L.D. Travis; Light Scattering by Nonspherical Particles: Theory, Measurements, and Applications, Academic Press, San Diego (1999).

[15] M. J. Mishchenko, L. D. Travis y A. A. Lacis; Scattering, Absorption, and Emission of Light by Small Particles, Cambridge University Press, Cambridge (2002).

[16] A. Doicu, T. Wriedt y Y. A. Eremin; Light Scattering by Systems of Particles, Springer, Berlin (2006).
[17] T. Rother y M. Kahnert; Electromagnetic Wave Scattering on Nonspherical Particles, Basic Methodology and Simulations, 2nd. Edition, Springer, berlin (2014).

[18] J. H. Seinfeld y S. N. Pandis; Atmospheric Chemistry and Physics, From Air Pollution to Climate Change, Third Edition, Wiley, New York (2016).

[19] R.G. Grainger; Some Useful Formulae for Aerosol Size Distributions and Optical Properties, http://eodg.atm.ox.ac.uk/user/grainger/research

/aerosols.pdf (Visto 24 agosto 2016).

[20] C. Zender; Particle Size Distributions: Theory and Application to Aerosols, Clouds, and Soils, http://patarnott.com/pdf/SizeDistributions.pdf (versión 2002) y

http://dust.ess.uci.edu/facts/psd/psd.pdf (version 2015) (visto 5 de junio 2016).

[21] M. Born y E. Wolf; Principles of Optics, Pergamon Press; Londres (1980).

[22] R. D. Guenter; Modern Optics, Johm Wiley \& Sons, New York (1990).

[23] G. B. Arfken, H. J. Weber y F. E. Harris; Mathematical Methods for Physicsts, Sixth Edit., Elsevier, Academic Press, New York (2005).

[24] F. Enguehard; Mie Theory and the Discrete Dipole Approximation. Calculating Radiative Properties of Particulate Media, with Application to Nanoestructured Materials, en Thermal Nanosystems and Nanomaterials, Volz, S. (Editor), 151-212, Springer, New York (2009)

[25] W.T. Grandy; Scattering of Waves from Large Spheres, Cambridge University Press, Cambridge (2005).

[26] P. Laven; J. Nanophotonics 4, 1 (2010).

[27] M.J. Berg; J. Quantitative Spectroscopy \& Radiative Transfer 112, 1170 (2011).

[28] A. Lampado; Light Scattering by a Spherical Particle, http://www. scattport.org/index.php/programs-menu /mie-type-codes-menu/112-mie-mathematica-lampado (visto 12 de julio de 2016).

[29] Q. Fu y W. Sun; Applied Optics 40, 1354 (2002).

[30] Q. Fu y W. Sun; J. Quantitative Spectroscopy \& Radiative Transfer 100, 137 (2006). 